\title{
REVIEW
}

\section{Hepatosplenic and Muscular Sarcoidosis: Characterization with MR Imaging}

\author{
Tetsuro Sekine ${ }^{1 *}$, Yasuo Amano ${ }^{1}$, Fumitaka HidakA ${ }^{1}$, Ryo TAKagi ${ }^{1}$, \\ Tadashi MACHIDA ${ }^{1}$, Zenya NAITO ${ }^{2}$, and Shinichiro Kumita ${ }^{1}$ \\ Departments of ${ }^{1}$ Radiology and ${ }^{2}$ Pathology, Nippon Medical School \\ 1-1-5 Sendagi, Bunkyo-ku, Tokyo 113-8603, Japan \\ (Received December 13, 2011; Accepted February 9, 2012)
}

\begin{abstract}
Sarcoidosis is a multisystem disorder of unknown etiology that involves multiple organs. Computed tomography is the first-line imaging modality for diagnosing sarcoidosis because of its capacity to detect hilar lymphadenopathy and pulmonary lesions. Magnetic resonance (MR) imaging provides good soft tissue contrast that is useful for detecting sarcoidosis in some body parts, including skeletal muscle. Signal intensity on pre- and postcontrast $T_{1}$ - and $T_{2}$-weighted imaging may reflect disease activity and the pathological appearance of sarcoidosis. In this review, we demonstrate these conventional MR imaging findings of hepatosplenic and muscular sarcoidosis and describe the usefulness of diffusionweighted imaging for detecting sarcoidosis.
\end{abstract}

\section{Keywords: diffusion-weighted imaging, MRI, sarcoidosis}

\section{Introduction}

Sarcoidosis is a systemic disorder of unknown etiology characterized histologically by noncaseating epithelioid granulomas in the absence of pathogens. ${ }^{1}$ The disease most commonly involves the lung and lymph nodes, followed by the skin, eye, liver, spleen, nervous system, bone marrow, and heart. Sarcoidosis commonly appears before age 50 years, and its incidence peaks between ages 20 and 39 years. $^{2}$ The annual incidence of sarcoidosis is highest in northern European countries (5 to 40 cases per 100,000 people) and lower in Japan (one to 2 cases per 100,000 people). ${ }^{3}$ In the only population-based incidence study of sarcoidosis in the United States, the annual rates of systemic sarcoidosis were 5.9 per 100,000 for men and 6.3 per 100,000 for women. ${ }^{4}$

Often, sarcoidosis is first suspected when abnormalities involving the lung and intrathoracic lymph nodes are detected with chest radiography and computed tomography (CT). Sarcoidosis can show a wide variety of nonspecific clinical symptoms, organ involvement, disease duration, and severity, ${ }^{5}$ but bilateral hilar adenopathy on chest $\mathrm{CT}$ can strongly indicate its presence. Because $50 \%$ of symptomatic patients with sarcoidosis initially present

\footnotetext{
*Corresponding author, Phone: +81-3-5814-6240, Fax: + 81-
} 3-5685-1795, E-mail: tetsuro.sekine@gmail.com with extrathoracic involvement, we must know the imaging features of this disease. In particular, magnetic resonance (MR) imaging assessment of sarcoidosis should be undertaken in the body and extremities because its soft-tissue contrast is greater than that achieved with CT.

We describe the conventional MR imaging techniques available for assessing sarcoidosis and the MR imaging appearances of the disease in the liver, spleen, and skeletal muscle, discuss the differential diagnosis of sarcoidosis based on these findings, and demonstrate the usefulness of diffusion-weighted imaging (DWI) for detecting hepatosplenic and muscular sarcoidosis.

\section{MR Imaging Techniques for Diagnosing Sar- coidosis}

$\mathrm{T}_{1^{-}}$and $\mathrm{T}_{2}$-weighted $\mathrm{MR}$ imaging are routinely used to examine the organs of patients with suspected sarcoidosis. $\mathrm{T}_{2}$-weighted imaging identifies active inflammation as lesions of high intensity and fibrosis as lesions of low intensity, valuable information in assessing disease activity. Sarcoidosis can be treated with steroids and immunosuppressants. Short inversion-time inversion recovery (STIR) imaging can be used as an alternative to fat-suppressed $\mathrm{T}_{2}$-weighted imaging in the extremities, where magnetic inhomogeneity can lead to insufficient fat suppression or unwanted water suppres- 
sion by spectrally selective fat-suppression techniques. Contrast-enhanced $\mathrm{T}_{1}$-weighted imaging is valuable for detecting sarcoidosis in the brain and musculoskeletal lesions and for assessing disease activity.

Table 1 shows the MR imaging features that correspond to the various tissue components of sarcoidosis. Table 2 summarizes the MR imaging features of sarcoidosis in the liver, spleen, and skeletal muscle and conditions that mimic sarcoidosis.

\section{Hepatosplenic Sarcoidosis}

Of patients with systemic sarcoidosis, 24 to $94 \%$ have biopsy-documented hepatic sarcoidosis, including the 2 to $60 \%$ of patients with laboratoryevident liver dysfunction and the fewer than $5 \%$ of patients with symptomatic liver dysfunction. ${ }^{6}$ The spleen is more frequently involved than the liver in systemic sarcoidosis. Splenic involvement is confirmed in 24 to $59 \%$ of patients by biopsy $y^{7,8}$ and in 38 to $77 \%$ of patients by autopsy, ${ }^{9}$ and one previous study showed splenic lesions in $69 \%$ of patients with hepatic sarcoidosis. ${ }^{10}$

Table 1. Magnetic resonance (MR) imaging features corresponding to tissue components in sarcoidosis and its mimics

\begin{tabular}{cc}
\hline & MRI features \\
\hline active & hyperintense signal on $\mathrm{T}_{2} \mathrm{WI} / \mathrm{STIR} / \mathrm{DWI}$ \\
infiltration & strong Gd enhancement \\
fibrosis & hypointensity on $\mathrm{T}_{1} / \mathrm{T}_{2} \mathrm{WI}$ \\
& moderate Gd enhancement \\
\hline
\end{tabular}

DWI, diffusion-weighted imaging; Gd, gadolinium; STIR, short inversion-time inversion recovery; WI, weighted imaging. Sarcoidosis has 2 pathological stagesactive inflammation and fibrosis. $\mathrm{T}_{2}$-weighted and $\mathrm{Gd}$ enhanced $T_{1}$-weighted MR imaging may be valuable for differentiating the 2 components of sarcoidosis.
Hepatosplenic sarcoidosis most commonly manifests as organomegaly. ${ }^{11}$ Five to $15 \%$ of patients have multiple nodules in the liver and spleen observable on MR imaging (Fig. 1). ${ }^{12}$ Approximate lesion size in hepatosplenic involvement aids diagnosis. A relatively small lesion may suggest sarcoidosis, ${ }^{12}$ and when sarcoidosis involves the liver and spleen, splenic nodules are usually larger than hepatic lesions (Fig. 1A). ${ }^{12,13}$ Sarcoid nodules show hyper- or hypointensity on $\mathrm{T}_{2^{-}}$and diffusionweighted images (Fig. 1). Hyperintense lesions suggest the presence of inflammation, lymphoid cell infiltration, and edema, whereas hypointense lesions imply fibrosis and granulation.

Hepatosplenic sarcoidosis should be differentiated from other entities that demonstrate a nodular appearance in the liver or spleen. Liver abscess, liver tuberculosis, and Gamna-Gandy nodules can appear as multiple nodules that resemble sarcoid lesions (Fig. 2). The MR imaging features of the multiple and simultaneous involvement of the liver and spleen may help distinguish sarcoidosis from these mimics. Underlying conditions, such as liver cirrhosis and immunosuppression, may also narrow the differential diagnosis.

\section{Muscular Sarcoidosis}

Muscular sarcoidosis is observed in 50 to $80 \%$ of patients with systemic sarcoidosis, ${ }^{14}$ but most patients have no apparent symptoms. ${ }^{15}$ Elevated concentrations of muscle enzymes, such as creatine phosphokinase and myoglobin, usually occur in patients with muscular sarcoidosis. However, laboratory, CT, sonography, and gallium-67 scintigraphy findings are nonspecific.

Muscular sarcoidosis is most conspicuous on $\mathrm{T}_{2^{-}}$ weighted and contrast-enhanced $T_{1}$-weighted $M R$ imaging. Typical MR imaging findings of muscular sarcoidosis are "dark star" and "3 stripes" signs on $\mathrm{T}_{2}$ - and contrast-enhanced $\mathrm{T}_{1}$-weighted images. ${ }^{16}$

Table 2. Magnetic resonance (MR) imaging features of sarcoidosis and its mimics in each organ described in this report

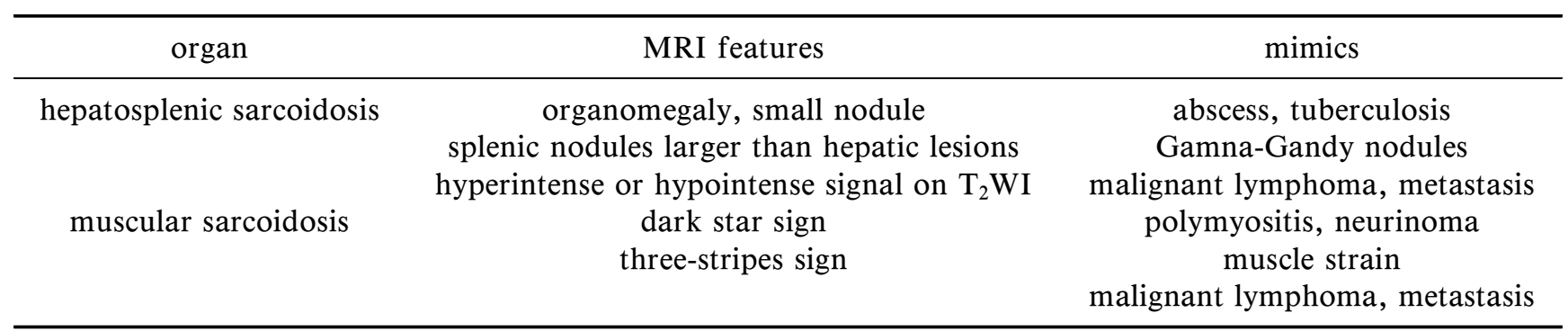

WI, weighted imaging 

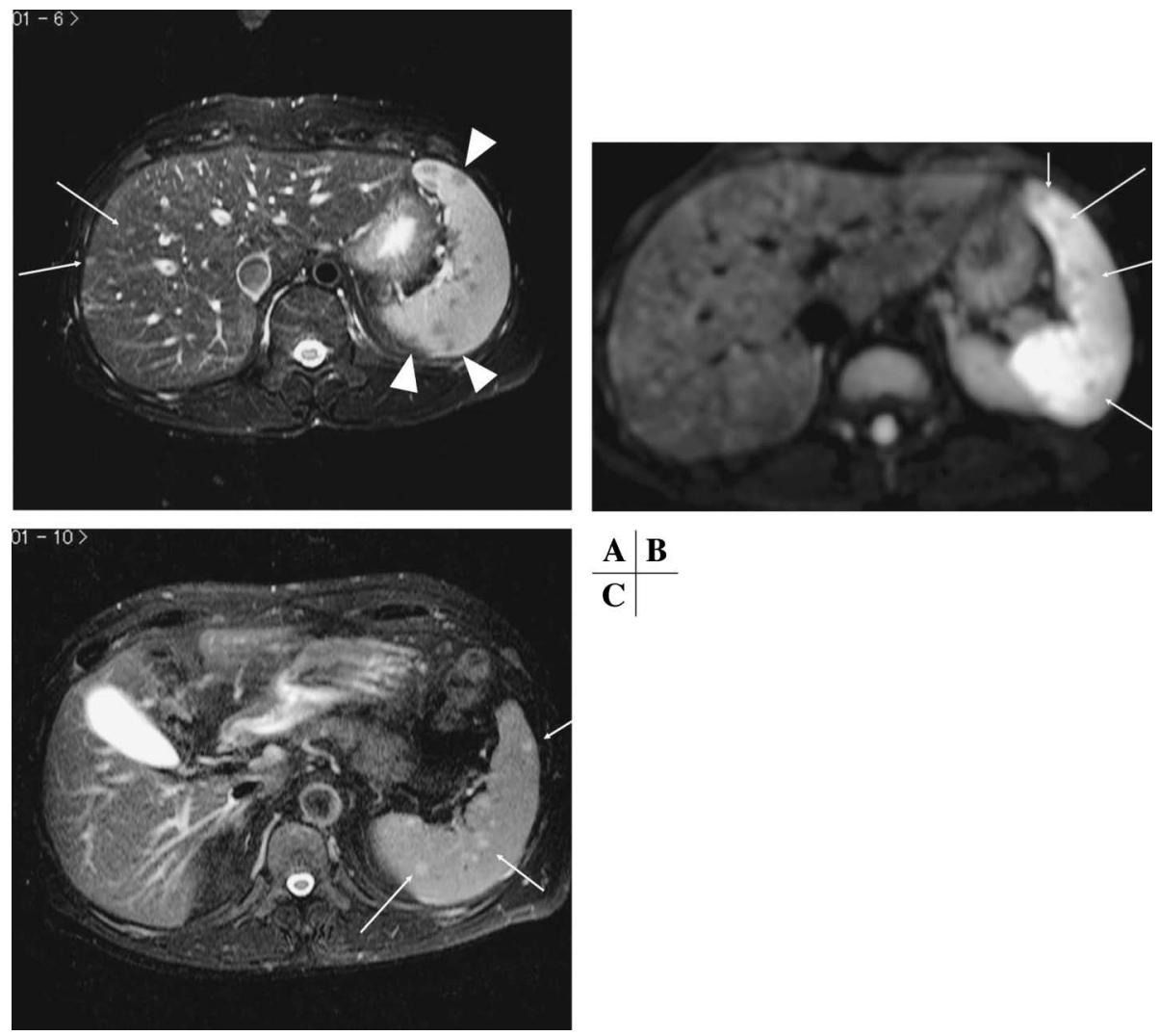

Fig. 1. (A) A 29-year-old man with hepatosplenic sarcoidosis. Transverse fatsuppressed $\mathrm{T}_{2}$-weighted images show hyperintense nodules in the liver (arrows), and splenic sarcoidosis shows low intensity on $\mathrm{T}_{2}$-weighted images (arrowheads). (B) Low intensity of splenic sarcoidosis on transverse diffusion- as well as $\mathrm{T}_{2}$-weighted images indicates fibrotic rather than inflammatory lesions (arrows). (C) A 42-year-old woman with splenic sarcoidosis. The splenic lesions show high signal intensity on fat-suppressed $\mathrm{T}_{2}$-weighted images (arrows).

Dark star sign is characterized by a star-shaped central structure with reduced signal intensity (Fig. $3 \mathrm{~A}, \mathrm{~B})$; diffusion-weighted images clearly visualize the presence of active inflammation in the muscle (Fig. 3C). The 3 stripes sign is usually observed on coronal and sagittal images and indicates inflammation extending along those muscle fibers, which appears as stripes (Fig. 3D). The center and inner stripe of muscular sarcoidosis shows low signal intensity on $\mathrm{T}_{2}$-weighted images because it consists of hyaline material and dense connective tissue resulting from longstanding inflammation. The peripheral and outer stripes show high signal intensity on $\mathrm{T}_{2}$-weighted and contrast-enhanced $\mathrm{T}_{1}$-weighted images, which may correspond to an active inflammatory granuloma containing epithelioid cells (Fig. 3E). ${ }^{17}$ Therefore, MR imaging is valuable for visualizing the extent of inflammation in patients diagnosed with muscular sarcoidosis and may be useful in assessing response to medical therapies, such as corticosteroid therapy.

Muscular sarcoidosis should be differentiated from muscular diseases such as polymyositis, neurinoma, and muscle strain (Fig. 4). The dark star sign or 3 stripes sign may be specific for sarcoidosis and useful in resolving the differential diagnosis.

\section{Diffusion-weighted Imaging for Detecting and Characterizing Sarcoidosis in the Liver, Spleen, and Muscle}

DWI, which is acquired with 2 high amplitude monopolar gradients, can be used in sarcoidosis. In the body region, the DWI signal is derived from the random motion of water molecules in the extracellular, intracellular, and intravascular spaces. ${ }^{18} \mathrm{Be}$ cause of the increased vascularity and cellularity of tumor tissues and inflammatory lesions, the contribution of intravascular water diffusion may account for a significant proportion of the MR signal. ${ }^{19,20}$ DWI is useful for detecting malignancies and evaluating their activity before and after treatment. ${ }^{21}$

Like $\mathrm{T}_{2}$-weighted imaging, DWI may be valuable 

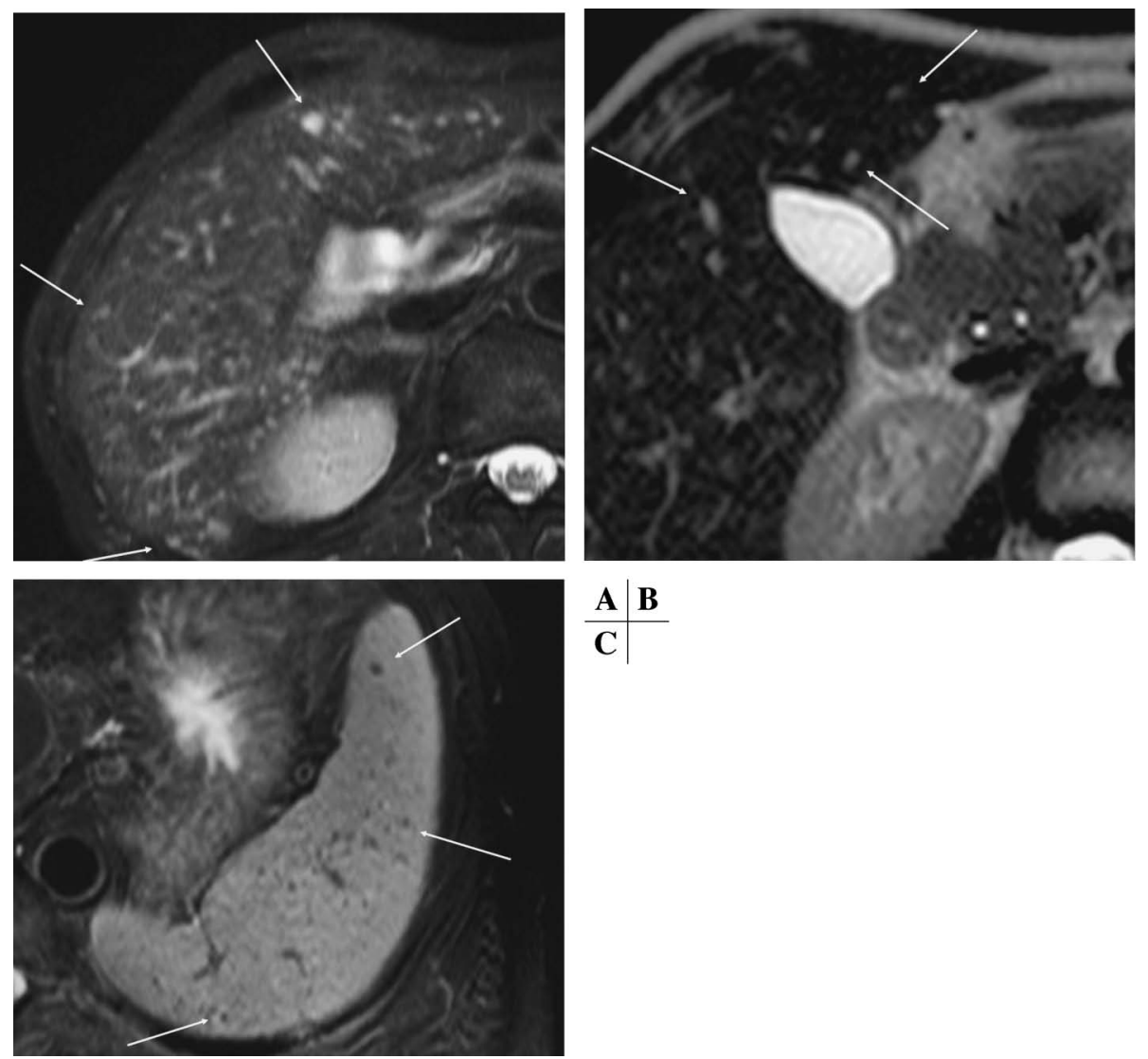

Fig. 2. Mimics of hepatosplenic sarcoidosis. (A) A 67-year-old man with liver abscess. These lesions appear as multiple hyperintense nodules in the liver on fat-suppressed $\mathrm{T}_{2}$-weighted images (arrow). (B) A 70-year-old woman with liver tuberculosis. Tuberculosis also appears as multiple hyperintense nodules in the liver on $\mathrm{T}_{2}$-weighted images (arrows). (C) A 65-year-old woman with GamnaGandy nodules in the spleen. Nodules show low signal intensity on fat-suppressed $\mathrm{T}_{2}$-weighted images because of their iron component (arrows).

for differentiating between 2 components of sarcoidosis, active infiltration and fibrosis. DWI demonstrates sarcoidosis with active infiltration involving the liver (Fig. 5A) and muscles as lesions of high intensity (Fig. 3C, E) and conspicuously detects small sarcoid nodules in the liver. The superior contrast between the normal hepatic parenchyma and sarcoidosis on DWI to that on $\mathrm{T}_{2}$-weighted (Fig. 5B) or contrast-enhanced $T_{1}$-weighted images indicates the potential advantage of DWI in visualizing inflammatory sarcoid lesions. Also like $\mathrm{T}_{2}$-weighted imaging, DWI may define sarcoidosis with fibrosis involving the spleen as lesions with low signal intensity (Fig. 1A, B). Warshauer and associates ${ }^{22}$ have suggested correspondence between the hypointense splenic nodules on $\mathrm{T}_{2}$-weighted images and the same degree of fibrosis associated with sarcoidosis. Because DWI has faster scan time and less blurring and does not require contrast agents, it can be used as a survey tool for systemic sarcoidosis.

Only a few reports describe the DWI findings of sarcoidosis. Kosucu and colleagues ${ }^{23}$ reported the high signal intensity of sarcoid lesions of the mediastinal lymph nodes on the apparent diffusion coefficient (ADC) map, and Okamoto and associates ${ }^{24}$ reported a case of neurosarcoidosis that showed marked hypointensity on DWI. We found that splenic sarcoidosis showed low signal intensity, whereas some hepatic and muscular sarcoidosis showed high signal intensity on DWI (Figs. 3C, 5A), findings discordant with the previous reports. Severity of inflammation, density of epithelioid cells, and disease location may contribute to the DWI signals and ADC values of sarcoidosis.

\section{Conclusions}

MR imaging is a valuable tool for detecting sarcoidosis in the liver, spleen, and skeletal muscles because it offers good soft-tissue contrast. We have reviewed the MR imaging techniques available for diagnosing sarcoidosis and the MR imaging find- 



\section{\begin{tabular}{l|l}
$\mathbf{A}$ & $\mathbf{B}$ \\
\hline $\mathbf{C}$ & $\mathbf{D}$
\end{tabular} \\ E}

Fig. 3. A 61-year-old man with muscular sarcoidosis. (A) Transverse $T_{2}$-weighted images and (B) gadolinium (Gd)-enhanced fat-suppressed $\mathrm{T}_{1}$-weighted images show the dark star sign (arrows). (C) Transverse diffusion-weighted images clearly visualize the presence of active inflammation in the muscle (arrows). (D) Coronal Gd-enhanced fat-suppressed $\mathrm{T}_{1}$-weighted images show the 3 stripes sign (arrows). (E) Muscle biopsy (original $\times 40$; hematoxylin-eosin stain) shows discrete noncaseating granulation with inflammatory cell infiltration.

ings of this disease, including hepatosplenic and muscular sarcoidosis. $T_{2}$-weighted, contrast-enhanced $\mathrm{T}_{1}$-weighted, and diffusion-weighted images may reflect the histological processes of sarcoidosis, including inflammation, edema, and fibrosis. Although some mimics are difficult to differentiate from sarcoidosis, awareness of the MR imaging findings of this disease can help narrow the differential diagnosis.

\section{References}

1. Hunninghake GW, Costabel U, Ando M, et al. ATS/ERS/WASOG statement on sarcoidosis. American Thoracic Society/European Respiratory Society/World Association of Sarcoidosis and other Granulomatous Disorders. Sarcoidosis Vasc Diffuse Lung Dis 1999; 16:149-173.

2. Rybicki BA, Major M, Popovich J Jr, Maliarik MJ, Iannuzzi MC. Racial differences in sarcoidosis 

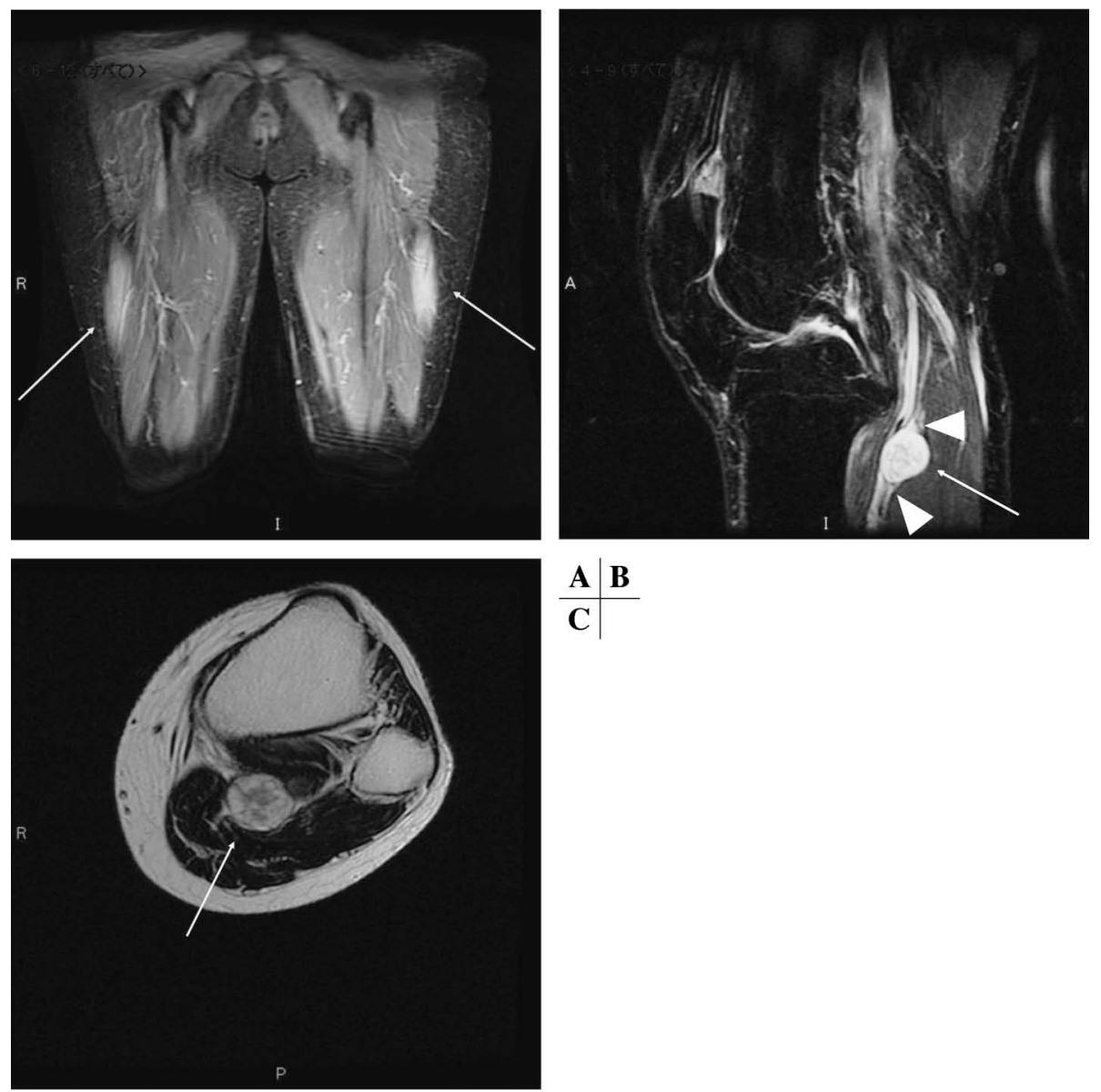

\section{\begin{tabular}{l|l}
$\mathbf{A}$ & $\mathbf{B}$ \\
\hline $\mathbf{C}$ &
\end{tabular}}

Fig. 4. Mimics of muscular sarcoidosis. (A) A 43-year-old woman with polymyositis. Coronal short inversion-time inversion recovery (STIR) image shows stripe-like hyperintensity, but it is not the " 3 stripes" pattern on fat-suppressed $\mathrm{T}_{2}$-weighted images (B). (C) A 52-year-old woman with neurinoma. Axial and sagittal $T_{2}$-weighted images show magnetic resonance (MR) imaging appearance similar to the dark star (arrow). However, sagittal STIR shows that neurinoma is present along the neurovascular bundle (arrow heads). This appearance is specific for neurinoma.

A
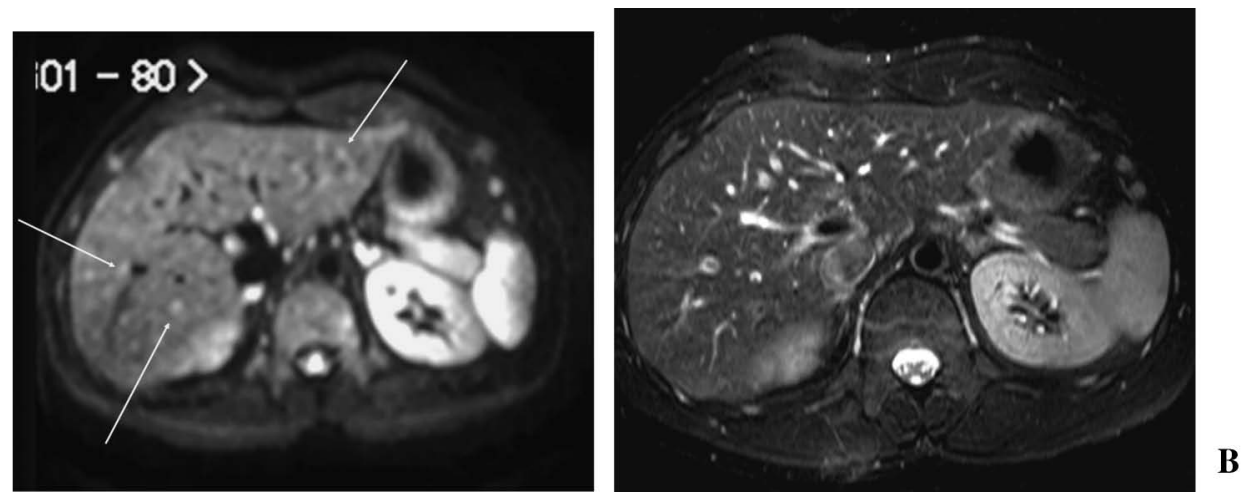

Fig. 5. A 45-year-old woman with hepatic sarcoidosis. (A) Transverse diffusion-weighted images show multiple hyperintense nodules in the liver (arrows). (B) These lesions cannot be detected on fat-suppressed $\mathrm{T}_{2}$-weighted images. 
incidence: a 5-year study in a health maintenance organization. Am J Epidemiol 1997; 145:234-241.

3. Iannuzzi MC, Rybicki BA, Teirstein AS. Sarcoidosis. N Engl J Med 2007; 357:2153-2165.

4. Henke CE, Henke G, Elveback LR, Beard CM, Ballard DJ, Kurland LT. The epidemiology of sarcoidosis in Rochester, Minnesota: a populationbased study of incidence and survival. Am J Epidemiol 1986; 123:840-845.

5. Nunes H, Brillet PY, Valeyre D, Brauner MW, Wells AU. Imaging in sarcoidosis. Semin Respir Crit Care Med 2007; 28:102-120.

6. Klatskin G. Hepatic granulomata: problems in interpretation. Ann N Y Acad Sci 1976; 278:427432.

7. Taavitsainen M, Koivuniemi A, Helminen J, et al. Aspiration biopsy of the spleen in patients with sarcoidosis. Acta Radiol 1987; 28:723-725.

8. Selroos O, Koivunen E. Usefulness of fine-needle aspiration biopsy of spleen in diagnosis of sarcoidosis. Chest 1983; 83:193-195.

9. Lynch JP 3rd, Sharma OP, Baughman RP. Extrapulmonary sarcoidosis. Semin Respir Infect 1998; 13:229-254.

10. Scott GC, Berman JM, Higgins JL Jr. CT patterns of nodular hepatic and splenic sarcoidosis: a review of the literature. J Comput Assist Tomogr 1997; 21:369-372.

11. Britt AR, Francis IR, Glazer GM, Ellis JH. Sarcoidosis: abdominal manifestations at CT. Radiology 1991; 178:91-94.

12. Warshauer DM, Molina PL, Hamman SM, et al. Nodular sarcoidosis of the liver and spleen: analysis of 32 cases. Radiology 1995; 195:757-762.

13. Koyama T, Ueda H, Togashi K, Umeoka S, Kataoka M, Nagai S. Radiologic manifestations of sarcoidosis in various organs. Radiographics 2004; 24:87-104.

14. Resnick D, Niwayama G. Sarcoid, In: Resnick D, eds. Diagnosis of bone and joint disorders. 3rd ed. Philadelphia, Pa: Saunders, 1995; 4333-4352.

15. Moore SL, Teirstein AE. Musculoskeletal sarcoidosis: spectrum of appearances at MR imaging. Radiographics 2003; 23:1389-1399.

16. Otake S. Sarcoidosis involving skeletal muscle: imaging findings and relative value of imaging procedures. AJR Am J Roentgenol 1994; 162:369-375.

17. Otake S, Banno T, Ohba S, Noda M, Yamamoto M. Muscular sarcoidosis: findings at MR imaging. Radiology 1990; 176:145-148.

18. Le Bihan D, Breton E, Lallemand D, Aubin ML, Vignaud J, Laval-Jeantet M. Separation of diffusion and perfusion in intravoxel incoherent motion MR imaging. Radiology 1988; 168:497-505.

19. Thoeny HC, De Keyzer F, Vandecaveye V, et al. Effect of vascular targeting agent in rat tumor model: dynamic contrast-enhanced versus diffusion-weighted MR imaging. Radiology 2005; 237 : 492-499.

20. Koh DM, Takahara T, Imai Y, Collins DJ. Practical aspects of assessing tumors using clinical diffusion-weighted imaging in the body. Magn Reson Med Sci 2007; 6:211-224.

21. Koh DM, Collins DJ. Diffusion-weighted MRI in the body: applications and challenges in oncology. AJR Am J Roentgenol 2007; 188:1622-1635.

22. Warshauer DM, Lee JK. Imaging manifestations of abdominal sarcoidosis. AJR Am J Roentgenol 2004; 182:15-28.

23. Kosucu P, Tekinbas C, Erol M, et al. Mediastinal lymph nodes: assessment with diffusion-weighted MR imaging. J Magn Reson Imaging 2009; 30: 292-297.

24. Okamoto K, Ito J, Ishikawa K, Sakai K, Tokiguchi S. Diffusion-weighted echo-planar MR imaging in differential diagnosis of brain tumors and tumorlike conditions. Eur Radiol 2000; 10:1342-1350. 